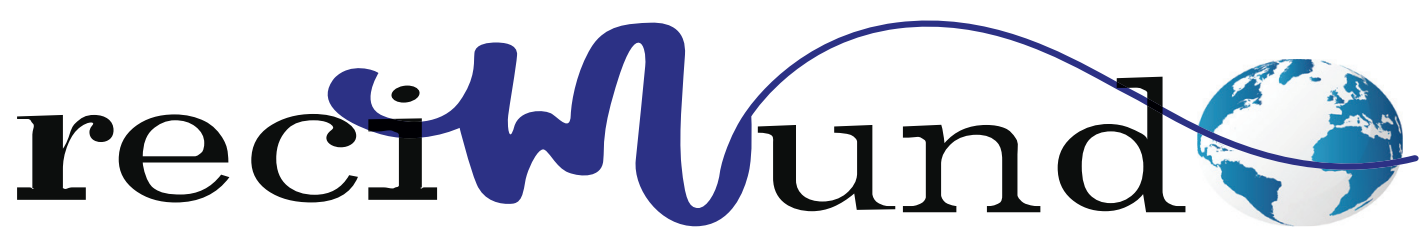

Revista Científica Mundo de la Investigación y el Conocimiento

DOI: 10.26820/recimundo/4.(2).mayo.2020.31-39

URL: http://recimundo.com/index.php/es/article/view/820

EDITORIAL: Saberes del Conocimiento

REVISTA: RECIMUNDO

ISSN: 2588-073X

TIPO DE INVESTIGACIÓN: Artículo de Revisión

CÓDIGO UNESCO: 3209 Farmacología;

3209.01 Análisis de Medicamentos

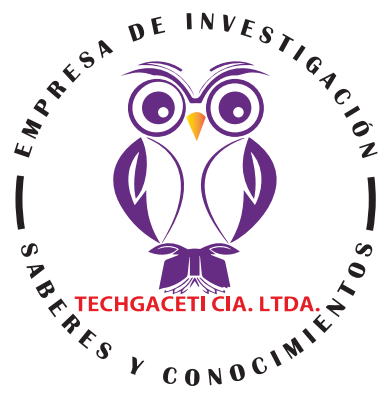

PAGINAS: 31-39

\title{
Recomendaciones de manejo farmacológico en COVID-19
}

Recommendations for pharmacological management in COVID-19

Recomendações para manejo farmacológico no COVID-19

Genesis Carolina Vargas Párraga; Franklin Fernando Chafla Pinduisaca²; Susy Arelis López Laaz;

Wilson Pascual Correa Quinto ${ }^{4}$

RECIBIDO: 15/03/2020 ACEPTADO: 20/03/2020 PUBLICADO: 20/05/2020

1. Médico; Investigador Independiente; Guayaquil, Ecuador; franceis.vargas@gmail.com; (iD https://orcid. org/0000-0002-9467-6479

2. Médico; Investigador Independiente; Guayaquil, Ecuador; drchafla@hotmail.com; (iD https://orcid.org/00000003-3599-0238

3. Médico; Investigador Independiente; Guayaquil, Ecuador; Slimed_992@hotmail.com; iD https://orcid.org/00000002-3981-9852

4. Médico; Investigador Independiente; Guayaquil, Ecuador; Willy_2293@hotmail.com; (iD https://orcid.org/00000002-6299-9644

\section{CORRESPONDENCIA}

Genesis Carolina Vargas Párraga

franceis.vargas@gmail.com

Guayaquil, Ecuador

○ RECIMUNDO; Editorial Saberes del Conocimiento, 2020 


\section{RESUMEN}

Durante el mes de diciembre del pasado 2019 fueron identificadas en Wuhan (China) una serie de casos de neumonía, los cuales estaban siendo originados por una nueva clase de coronavirus. Esta nueva clase de coronavirus ha adquirido diferentes denominaciones, tales como: 2019-nCoV nombrado así por la Organización Mundial de la Salud (OMS) y SARS-CoV-2 denominación concedida por el Comité Internacional de Taxonomía de Virus. La enfermedad que causa este virus se ha denominado 2019-nCoV. Hasta el 4 de mayo del 2020 la OMS había registrado en todo el mundo un total de 1.477 .448 casos confirmados, un total de 86.342 muertos y el virus presente en los 54 países, zonas y territorios contados con fines epidemiológicos. La realidad actual es que no existe ningún tratamiento farmacológico específico para el tratamiento COVID-19 cuya eficacia y seguridad se encuentre demostrada. El propósito fundamental del presente estudio es plasmar el tratamiento farmacológico de la COVID 19 que se maneja a nivel internacional. El diseño de investigación que se llevó a cabo es de tipo documental o bibliográfico. Los medicamentos más usados en todo el mundo para el tratamiento de la COVID 19 son: Hidroxicloroquina, Remdesivir, Lopinavir-Ritonavir, Interferón, Oseltamivir, Arbidol, Favipiravir y Vitamina C, entre otros. Es fundamental contribuir todos juntos como sociedad en la contención de este virus para ganar tiempo en la investigación de fármacos que garanticen un tratamiento efectivo y de vacunas que permitan su prevención, y esperamos que estas revisiones literarias contribuyan a seguir tratando de la mejor manera posible a los infectados con COVID-19.

Palabras clave: Tratamiento, Farmacológico, Coronavirus, Covid-19, Medicamentos.

\section{ABSTRACT}

During the month of December last 2019, a series of cases of pneumonia were identified in Wuhan (China), which were caused by a new class of coronavirus. This new class of coronavirus has acquired different names, such as: 2019-nCoV named after the World Health Organization (WHO) and SARS-CoV-2, name given by the International Committee on Virus Taxonomy. The disease causing this virus has been named 2019-nCoV. As of May 4, 2020, the WHO had registered a total of 1,477,448 confirmed cases worldwide, a total of 86,342 deaths and the virus present in the 54 countries, areas and territories counted for epidemiological purposes. The current reality is that there is no specific pharmacological treatment for COVID-19 treatment whose efficacy and safety have been demonstrated. The fundamental purpose of this study is to capture the pharmacological treatment of COVID 19 that is managed internationally. The research design that was carried out is documentary or bibliographic. The most widely used drugs worldwide for the treatment of COVID 19 are: Hydroxychloroquine, Remdesivir, Lopinavir-Ritonavir, Interferon, Oseltamivir, Arbidol, Favipiravir and Vitamin C, among others. It is essential to contribute together as a society in the containment of this virus to save time in the investigation of drugs that guarantee effective treatment and vaccines that allow its prevention, and we hope that these literary reviews will contribute to continue treating in the best possible way to those infected with COVID-19.

Keywords: Treatment, Pharmacological, Coronavirus, Covid-19, Medications.

\section{RESUMO}

Durante o mês de dezembro de 2019, foram identificadas uma série de casos de pneumonia em Wuhan (China), causados por uma nova classe de coronavírus. Essa nova classe de coronavírus adquiriu nomes diferentes, como: 2019-nCoV com o nome da Organização Mundial de Saúde (OMS) e SARS-CoV-2, nome dado pelo Comitê Internacional de Taxonomia de Vírus. A doença que causa esse vírus foi denominada 2019-nCoV. Em 4 de maio de 2020, a OMS registrou um total de 1.477.448 casos confirmados em todo o mundo, um total de 86.342 mortes e o vírus presente nos 54 países, áreas e territórios contados para fins epidemiológicos. A realidade atual é que não há tratamento farmacológico específico para o tratamento com COVID-19 cuja eficácia e segurança foram demonstradas. O objetivo fundamental deste estudo é capturar o tratamento farmacológico do COVID 19 gerenciado internacionalmente. O desenho da pesquisa realizado é documental ou bibliográfico. Os medicamentos mais amplamente utilizados no mundo para o tratamento de COVID 19 são: hidroxicloroquina, remdesivir, lopinavir-ritonavir, interferon, oseltamivir, arbidol, favipiravir e vitamina C, entre outros. É essencial contribuir em conjunto, como sociedade, na contenção deste vírus, para economizar tempo na investigação de medicamentos que garantam tratamento eficaz e vacinas que permitam sua prevenção, e esperamos que essas revisões literárias contribuam para continuar o tratamento da melhor maneira possível. caminho para aqueles infectados com COVID-19.

Palavras-chave: Tratamento Farmacológico, Coronavírus, Covid-19, Medicamentos. 


\section{Introducción}

Durante el mes de diciembre del pasado 2019 fueron identificadas en Wuhan (China) una serie de casos de neumonía los cuales estaban siendo originados por una nueva clase de coronavirus. Esta nueva clase de coronavirus ha adquirido diferentes denominaciones, tales como: 2019-nCoV nombrado así por la OMS y SARS-CoV-2 denominación concedida por el Comité Internacional de Taxonomía de Virus. La enfermedad que causa este virus se ha denominado 2019-nCoV. Este nuevo coronavirus fue anunciado oficialmente el 7 de enero de 2020, por las autoridades chinas como el agente que ocasionó dichas neumonías. (Palacios, Santos, Velázquez, \& León, 2020)

En la actualidad se ha registrado una rápida propagación a escala comunitaria, regional e internacional, de esta nueva enfermedad, con un incremento exponencial del número de casos y muertes. Para el 30 de enero del 2020, el Director General de la OMS en sus declaraciones, declaró al COVID-19 como una emergencia de salud pública, de gran relevancia internacional, conforme con el Reglamento Sanitario Internacional (2005). El primer caso confirmado en la Región de las Américas, se registró en los Estados Unidos, en fecha 20 de enero del 2020, mientras que Brasil reportó el primer caso correspondiente a América Latina y el Caribe, en fecha 26 de febrero del 2020. El COVID-19 ha partir de ese momento se ha propagado a todos los 54 países y territorios de la Región de las Américas. Hasta el 4 de mayo del 2020 la Organización Mundial de la Salud había registrado un total de 1.477.448 casos confirmados, un total de 86.342 muertos y el virus presente en los 54 países, zonas y territorios contados con fines epidemiológicos. (Organización Panamericana de la Salud, 2020)

Esta pandemia por coronavirus SARS-CoV-2 (COVID-19) plantea a todos los niveles (internacional, nacional y regional) nuevos retos en la gestión sanitaria, que en la actualidad presenta múltiples incertidumbres. Uno de estos retos más resaltantes se refiere a la terapia farmacológica más idónea que beneficie la curación de los pacientes contagiados y para prevenir la infección en la población que se encuentra sana. La realidad actual es que no existe ningún tratamiento farmacológico específico para el tratamiento COVID-19 cuya eficacia y seguridad se encuentre demostrada en estudios de calidad contrastada. Sin embargo, existen algunas alternativas propuestas desde un enfoque experimental con la finalidad de que sean útiles en el tratamiento de esta enfermedad. (Saiz Fernández, 2020)

Con una cantidad tan grande de contagios y muertes y una distribución mundial tan extensa, el desconocimiento general de muchos aspectos tanto del virus como de la enfermedad y la premura por encontrar soluciones que normalicen la salud de la población mundial, ha llevado a los investigadores y las agencias de salud pública a que en lugar de crear compuestos desde cero, proceso que puede tardar años desarrollar y probar, se encuentran en la búsqueda de reutilizar medicamentos ya aprobados para otras enfermedades y de los cuales se sabe que son, en gran medida seguros. Asimismo, se encuentran analizando medicamentos no aprobados que han funcionado bien en estudios con animales y usados contra los otros dos coronavirus mortales. (Kupferschmidt \& Cohen, 2020)

El presente trabajo plasma los aspectos más resaltantes en cuanto al tratamiento farmacológico del COVID 19 con la finalidad de reunir la opinión de algunos autores en base a sus estudios e informaciones más actualizadas de las principales agencias de salud pública mundial.

\section{Materiales y Métodos}

Para el desarrollo del presente estudio se llevó a cabo una búsqueda con el propósito

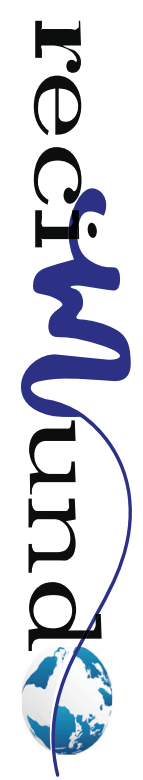


de recopilar y seleccionar el material bibliográfico digitalizado que permitió desarrollar el tema del tratamiento farmacológico del COVID 19. En virtud de lo cual, se clasifica la investigación llevada a cabo como de tipo documental o bibliográfica.

El material digitalizado fue ubicado por medio de bases de datos y páginas web relacionadas con el área de la salud mundial, entre las que se encuentran: MedlinePlus, PubMed, SciELO, Organización Panamericana de la Salud, Organización Mundial de la Salud, entre otras. Para la búsqueda se utilizaron los siguientes descriptores: "Covid 19", "Coronavirus"; "tratamiento del covid 19" y "tratamiento farmacológico del covid 19". La búsqueda arrojó aproximadamente un centenar de miles de registros bibliográficos, los cuales posteriormente fueron filtrados atendiendo a los criterios de idioma los cuales fueron español e inglés, relevancia del tema, con una correlación temática y actuales (fecha de publicación en los últimos cuatro años).

\section{Resultados}

La Organización Mundial de la Salud (2020) ha dejado claro que en la actualidad no existe ningún medicamento autorizado para el tratamiento o la prevención de la COVID-19. Aclaran que, hasta la fecha, no es recomendable ningún medicamento específico para la prevención o el tratamiento de la infección por el nuevo coronavirus (2019nCoV). No obstante, es fundamental atender adecuadamente a las personas contagiadas por este virus a los fines de aliviar y tratar la sintomatología que se presente y administrar las medidas de apoyo optimizadas a aquellos pacientes que presenten síntomas graves. Asimismo, se encuentra en estudio algunos tratamientos específicos que se probarán en ensayos clínicos.

En este orden de ideas, el tratamiento farmacológico se indica en base a medicamentos usados para otras enfermedades y que dada su composición estos podrían ser útiles en el tratamiento de este nuevo coronavirus. Una característica común de los medicamentos utilizados para el Covid 19 hasta la actualidad, es que la mayoría de estos medicamentos son antivirales y en muchos países han reportado su efectividad.

La OMS se encuentra dirigiendo el estudio de cuatro tratamientos de coronavirus más prometedores, se trata de una combinación de drogas usadas ya en contra el VIH. La OMS "anunció un gran ensayo global, Ilamado SOLIDARIDAD, para descubrir si alguno puede tratar las infecciones con el nuevo coronavirus para la peligrosa enfermedad respiratoria". En vista de este estudio algunos científicos han sugerido docenas de compuestos que ya existen en el mercado para la prueba, sin embargo la OMS se centrará en las cuatro terapias más prometedoras: un compuesto antiviral experimental llamado remdesivir, los medicamentos contra la malaria cloroquina e hidroxicloroquina, una combinación de dos medicamentos contra el $\mathrm{VIH}$, lopinavir y ritonavir y esa misma combinación más interferón beta, el cual es un mensajero del sistema inmune que puede colaborar en lisiar a los virus. (Kupferschmidt \& Cohen, 2020)

En atención a estos cuatro fármacos propuestos para la investigación por la OMS y en base a otros usados con éxito en el tratamiento del COVID 19 y respaldados por estudios de carácter científico, es que se desarrollará la presente revisión. A continuación, algunos de los principales fármacos usados a nivel mundial en el tratamiento del COVID 19:

\section{Hidroxicloroquina}

La hidroxicloroquina pertenece a la tipología de medicamentos nombrados antimaláricos. Es usada en la prevención y tratamiento de los ataques agudos de malaria. Asimismo, se utiliza para el tratamiento del 
lupus eritematoso sistémico y discoide, así como para tratar la artritis reumatoide en aquellos pacientes cuyos síntomas persisten pese a otros tratamientos. (Sociedad Americana de Farmacéuticos Institucionales SA, 2016)

Actualmente, la hidroxicloroquina se está usando en ensayos clínicos relacionados con el tratamiento del síndrome de inmunodeficiencia adquirida (SIDA). Recientemente se usó en un ensayo con pacientes para el tratamiento del COVID-19, el cual arrojó como resultado que el $100 \%$ de los pacientes tratados con este fármaco "en combinación con la azitromicina antibiótica macrólida se curaron virológicamente en comparación con el $57.1 \%$ en pacientes tratados con hidroxicloroquina sola, y $12.5 \%$ en el grupo control". (Rosa \& Santos, 2020)

Saiz Fernández, en su estudio relacionado con la consideración de eventos adversos graves en la prolongación de los tratamientos del COVID 19, específicamente de la revisión del estudio de Gautret et al, quien administró hidroxicloroquina para el tratamiento de esta enfermedad, concluyó:

- Actualmente no existe un tratamiento efectivo bien validado para el COVID-19.

- La cloroquina/hidroxicloroquina ha mostrado eficacia in vitro frente al COVID-19 pero no hay ensayos clínicos que corroboren su utilidad en la práctica clínica.

- El estudio de Gautret et al., a partir de 36 pacientes, ha mostrado una reducción significativa con hidroxicloroquina en la carga viral del COVID-19 a los 6 días de la inclusión. La adición de azitromicina se ha asociado a resultados aún más positivo.

- Es preciso considerar la posibilidad de eventos adversos graves (prolongación del intervalo QT, arritmias) asociados al uso de hidroxicloroquina y azitromicina.

- El uso masivo de estos fármacos fuera de indicación para el COVID-19 puede poner en riesgo el suministro ordinario para la malaria, lupus o artritis reumatoide. (Saiz Fernández, 2020)

Por otra parte, la OMS (2020) refiere que a pesar de que están en marcha varios ensayos de medicamentos, hasta el momento no se ha demostrado que la hidroxicloroquina ni ningún otro fármaco puedan curar o prevenir la COVID-19. El uso indebido de la hidroxicloroquina puede provocar graves efectos secundarios y problemas de salud e incluso causar la muerte.

\section{Remdesivir}

Remdesivir es un inhibidor análogo de nucleótidos de la ARN-polimerasa dependiente de ARN EBOV. Dyer y col. 2019, referidos por Rosa \& Santos (2020) "describieron los hallazgos preliminares de una tasa de mortalidad del 33\% en 499 pacientes tratados con remdesivir contra la enfermedad por EBOV en las primeras etapas de la infección". Asimismo, estos autores observaron una la tasa de mortalidad del $75 \%$ de pacientes infectados no tratados durante el mismo período epidémico.

Por otra parte, Serrano (2020) refiere que el Remdesivir el cual fue diseñado originalmente para el tratamiento del ébola, no mostró ser efectivo. No obstante, parece tener potencial en el tratamiento de los coronavirus en base a pruebas de células cultivadas en laboratorios. Igualmente, existen reportes anecdóticos de que este tratamiento ha sido beneficioso en pacientes con covid-19, sin embargo, no resulta suficiente con el propósito de aseverar que la droga es efectiva.

Por otro lado, a fines de abril 2020, la Administración de Drogas y Alimentos (FDA) de los Estados Unidos emitió una aprobación de emergencia para el fármaco remdesivir a objeto de que se usara en el tratamiento de pacientes gravemente enfermos con Covid-19. "EI FDA se apresuró a aprobar remdesivir bajo disposiciones de uso de emer-

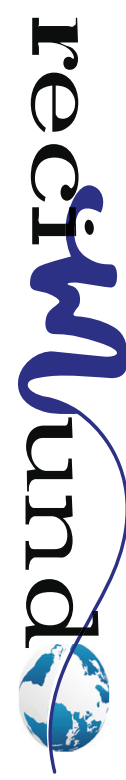


gencia, después de que un ensayo federal demostró mejoras modestas en pacientes gravemente enfermos". (Kolata, 2020)

\section{Lopinavir-Ritonavir}

La combinación de lopinavir y ritonavir es usada junto con otros medicamentos con la finalidad de tratar el virus de inmunodeficiencia humana $(\mathrm{VIH})$. Lopinavir y ritonavir son del grupo de los medicamentos nombrados como inhibidores de la proteasa. Su efecto se produce al reducir la cantidad de $\mathrm{VIH}$ en la sangre. Al tomarse juntos ambos medicamentos, lopinavir y ritonavir, colaboran en aumentar la cantidad de lopinavir en el cuerpo a objeto de que el medicamento tenga un mayor efecto. "A pesar de lopinavir y ritonavir no cura el $\mathrm{VIH}$, estos medicamentos pueden disminuir la probabilidad de desarrollar síndrome de inmunodeficiencia adquirida (SIDA) y enfermedades asociadas al $\mathrm{VIH}$, como infecciones graves o cáncer". (Sociedad Americana de Farmacéuticos Institucionales SA, 2016)

Quedando claro el uso habitual en varios países de la combinación de lopinavir-ritonavir en el tratamiento del SIDA, es importante resaltar su importancia para el tratamiento del COVID 19. Ambas drogas son inhibidores de la proteasa del VIH, pero ritonavir también es un inhibidor de citocromo P450 y GP, un hecho que respalda las actividades farmacocinéticas y farmacodinámicas de lopinavir contra el VIH. Dicha combinación, más el interferón b-1b, se encuentra en la fase 2 para el tratamiento con MERS. (Rosa \& Santos, 2020)

Bin Cao et al. (2020) acerca del uso de lopinavir-ritonavir en el tratamiento del COVID 19, realizaron el siguiente estudio:

Un ensayo aleatorizado, controlado y abierto que incluyó a pacientes adultos hospitalizados con infección confirmada por SARS-CoV-2, que causa la enfermedad respiratoria Covid-19 y una saturación de oxígeno (Sao2) del 94\% o menos mientras respiraban a temperatura ambiente. aire o una relación de la presión parcial de oxígeno (Pao2) a la fracción de oxígeno inspirado (Fio2) de menos de $300 \mathrm{~mm} \mathrm{Hg}$. Un total de 199 pacientes con infección por SARSCoV-2 confirmada por laboratorio se sometieron a aleatorización; 99 fueron asignados al grupo de lopinavir-ritonavir, y 100 al grupo de atención estándar. Los autores concluyeron: en pacientes adultos hospitalizados con Covid-19 grave, no se observó ningún beneficio con el tratamiento con lopinavir-ritonavir más allá de la atención estándar. En conclusión, encontraron que el tratamiento con lopinavir-ritonavir no aceleró significativamente la mejoría clínica, redujo la mortalidad ni disminuyó la detección de ARN viral de la garganta en pacientes con Covid-19 grave. Estos datos iniciales deberían informar futuros estudios para evaluar este y otros medicamentos en el tratamiento de la infección por SARS-CoV-2. Queda por determinar si combinar lopinavir-ritonavir con otros agentes antivirales, como se ha hecho en SARS5,20 y se está estudiando en MERS-CoV podría aumentar los efectos antivirales y mejorar los resultados clínicos.

\section{Interferón}

Gomez, Jimbo, \& Sánchez (2020), refieren acerca del interferón (INF) que se trata de un fármaco antineoplásico biológico, que se usa en el tratamiento de múltiples neoplasias hematológicas y enfermedades cutáneas. Descubierto en 1957 en Inglaterra, por un virólogo suizo llamado Jean Lindenmannace. Se encuentran varios tipos de interferón en base a sus acciones inmunomoduladoras y antivirales. En la actualidad el INF cuenta con la aprobación de la Food and Drug Administration (FDA) de los Estados Unidos para 6 indicaciones específicas: El sarcoma de kaposi (SK) relacionado al VIH, la hepatitis B, el condiloma acuminado, el linfoma folicular, la leucemia por células peludas y el melanoma. Asimismo, estos autores, en abril de este año realizaron una 
búsqueda de la literatura relacionada con estudios científicos, con la finalidad de dar respuesta a la interrogante: ¿Cuál es la eficacia del Interferón en el tratamiento de los pacientes con diagnóstico de COVID-19?, donde concluyeron que no existe evidencia clínica en humanos acerca de la acción del interferón en las infecciones por los coronavirus SARS y MERS. Las propuestas farmacológicas recientes para el tratamiento de la infección por SARS-CoV-2 se extrapolan de estos hallazgos in vitro. Las terapias de apoyo junto al uso de antivirales en la regulación inmune son importantes a tomarse en cuenta, principalmente en aquellos pacientes que se encuentran en estado grave y crítico, en estos podría mejorar la respuesta clínica y en algunos casos podría colaborar en evitar lesiones pulmonares residuales. (p. 1-5)

Los interferones (IFN) son proteínas que se unen a los receptores de las superficies celulares e inician cascadas de señalización JAK-STAT, con regulación transcripcional de genes controlados por interferones y efectos contra algunos virus como el virus de la hepatitis B y el virus de la hepatitis C. Se describe que el interferón humano recombinante $a 2 b$ tiene efectos inhibitorios sobre MERS-CoV y SARS-CoV, y el propósito de los ensayos clínicos encontrados para este trabajo es evaluar la eficacia y seguridad del interferón humano recombinante a2b en el tratamiento de pacientes con nueva infección por coronavirus. (Rosa \& Santos, 2020)

\section{Oseltamivir}

El oseltamivir es otro medicamento aprobado para el tratamiento de la gripe $A$ y $B$; inhibe la neuramina-idasa viral y, en consecuencia, bloquea la liberación de partículas virales de las células huésped, reduciendo la propagación en el tracto respiratorio. Además, el uso de oseltamivir ya se informó durante la epidemia de COVID-19 en China, con o sin antibióticos y corticosteroides.
El oseltamivir también se usa en un ensayo clínico con múltiples combinaciones con cloroquina y favipiravir, un análogo de nucleósido que es bien conocido como un medicamento antiviral de amplio espectro; ha mostrado una CE50 de $61.88 \mu \mathrm{M}$ contra SARS-CoV-2 y baja toxicidad (CC50> 400 $\mu \mathrm{M})$. (Rosa \& Santos, 2020)

\section{Arbidol y Favipiravir}

El Arbidol y el Favipiravir están entre los medicamentos recomendados por la Organización Mundial de la Salud para el tratamiento de la COVID 19.

Chen et al. (2020) Ilevaron a cabo un estudio acerca del Arbidol en el tratamiento del COVID 19, en el que refieren lo siguiente:

Realizamos un ensayo prospectivo, aleatorizado, controlado, abierto y multicéntrico. con pacientes adultos con COVID-19. Los pacientes fueron asignados aleatoriamente en un 1: 1 relación para recibir terapia convencional más Umifenovir (Arbidol) $(200$ mg * 3 / día) o Favipiravir (1600 mg * 2 / primer día seguido de 600 mg * 2 / día) durante 10 días. El primer resultado fue la tasa de recuperación clínica del día 7. Latencia al alivio de la pirexia y la tos, la tasa de oxigenoterapia auxiliar (AOT) o ventilación mecánica no invasiva (NMV) fueron los resultados secundarios. Los datos de seguridad fueron recolectados durante 17 días. Conclusiones: En comparación con Arbidol, Favipiravir no mejora significativamente la tasa de recuperación clínica del día 7. El favipiravir se asocia con una latencia significativamente acortada para el alivio de pirexia y tos. Los efectos adversos asociados a los antivirales de Favipiravir son leves y manejables. ( $p$. $7,18)$

\section{Vitamina C}

Finalmente, la vitamina $\mathrm{C}$ tiene actividad antioxidante y puede reducir el estrés oxidativo y la inflamación, efectos que mejoran la síntesis de vasopresores, mejoran la función 
de las células inmunes, mejoran la función endovascular y proporcionan modificaciones inmunológicas epigenéticas. Los ensayos clínicos han demostrado datos prometedores sobre la mejora de la mortalidad en la sepsis, pero se necesitan estudios más extensos para validar estas conclusiones. (Rosa \& Santos, 2020)

\section{Conclusiones}

En la actualidad la COVID 19 es una enfermedad desconocida, así el virus que la ocasiona, pero que se ha propagado por todo el mundo causando altos índices de contagios y muertes. Asimismo, esta pandemia ha repercutido negativamente en la humanidad causando no sólo daño en nuestra salud y pérdidas humanas, sino también atacando nuestra economía y el aparato productivo de los países del mundo que la padecen, disminuyendo la seguridad y las esperanzas de las personas más afectadas. Es mucho lo que se desconoce del virus, por lo tanto, son muchas las carencias e incertidumbres que nos rodean. Una de ellas es el tratamiento efectivo de la COVID 19.

Actualmente, se encuentra colaborando científicos de todo el mundo, así como especialistas en el área de salud pública, con la finalidad de unir esfuerzos e intercambiar datos científicos que aporten mayores conocimientos en el comportamiento del virus y la enfermedad.

Una de las respuestas a esta incertidumbre ha sido la aplicación de algunos fármacos ya existentes y efectivos en el tratamiento de otras infecciones, los cuales se han ido probando de manera empírica, obteniéndose registros en todo el mundo de casos en los que han resultado beneficiosos para los pacientes contagiados, ya sea de manera individual o combinados. Los medicamentos más usados en todo el mundo para el tratamiento de la COVID son: Hidroxicloroquina, Remdesivir, Lopinavir-Ritonavir, Interferón, Oseltamivir, Arbidol, Favipiravir y
Vitamina $\mathrm{C}$, entre otros.

Es fundamental contribuir todos juntos como sociedad en la contención de este virus para ganar tiempo en la investigación de fármacos que garanticen un tratamiento efectivo y de vacunas que permitan su prevención, y esperamos que estas revisiones literarias contribuyan a seguir tratando de la mejor manera posible a los infectados con COVID-19.

\section{Bibliografía}

Bin Cao, M., Yeming Wang, M., Danning Wen, M., Wen Liu, M., \& al., e. (19 de Marzo de 2020). Lopinavir-ritonavir en adultos hospitalizados con COVID-19 grave. Intramed. doi:10.1056/NEJMoa2001282

Chen, C., Zhang, Y., Huang, J., Yin, P., Cheng, Z., Wu, J., . . . Wang, X. (2020). Favipiravir versus Arbidol for COVID-19: A Randomized Clinical. medrxiv, 27. Recuperado el 10 de Mayo de 2020, de https://www.medrxiv.org/content/10.1101/2020.03.17.20037432v4.full.pdf

Gomez, A. M., Jimbo, R., \& Sánchez, X. (2020). Interferón en el tratamiento de la infección por COVID-19. Pontificia Universidad Católica del Ecuador (PUCE), 1-5. Recuperado el 05 de Mayo de 2020, de https://www.researchgate.net/publication/340680412

Kolata, G. (01 de Mayo de 2020). How Remdesivir, New Hope for Covid-19 Patients, Was Resurrected. The New York Times. Recuperado el 01 de Mayo de 2020, de https://www.nytimes.com/2020/05/01/ health/coronavirus-remdesivir.html

Kupferschmidt, K., \& Cohen, J. (25 de MArzo de 2020). OMS: megatrial de los cuatro tratamientos para COVID19 más prometedores para COVID19 más prometedores. Intramed. doi:10.1126/science.abb8497

OMS. (10 de Abril de 2020). Organización Mundial de la Salud. Recuperado el 01 de Mayo de 2020

Organización Panamericana de la Salud. (2020). Covid 19. Respuesta de la OPS/OMS. Organización Mundial de la Salud, Washintong D.C. Recuperado el 13 de Mayo de 2020, de file:///C:/Users/ Usuario/Desktop/aymee/PAHO-reporte-operacional-04.05.20.pdf

Palacios, M., Santos, E., Velázquez, M., \& León, M. (04 de Marzo de 2020). COVID-19, una emergencia de salud pública mundial. Rev Clin Esp., 1-7. 
Recuperado el 10 de Mayo de 2020, de https://doi. org/10.1016/j.rce.2020.03.001

Rosa, S. G., \& Santos, W. C. (2020). Ensayos clínicos de reposicionamiento de medicamentos para el tratamiento de la COVID-19. Revista Panamericana de la Salud Pública, 44, 1-7. doi:https://doi. org/10.26633/RPSP.2020.40

Saiz Fernández, L. C. (2020). Es preciso considerar la posibilidad de eventos adversos graves (prolongación del terapias frente al COVID-19). Sección de Innovación y Organización. SNS-O, Navarra. Recuperado el 05 de Mayo de 2020, de https://es. cochrane.org/sites/es.cochrane.org/files/public/ uploads/COVID-19/cochrane_navarra_covid-19_ cloroquina_hidroxicloroquina.pdf
Serrano, C. (24 de Marzo de 2020). Coronavirus: cuáles son los 4 tratamientos que la OMS estudia para combatir el covid-19. BBC News Mundo. Recuperado el 04 de Mayo de 2020

Sociedad Americana de Farmacéuticos Institucionales SA. (15 de Enero de 2016). MedlinePlus. Recuperado el 10 de Mayo de 2020, de https://medlineplus.gov/spanish/druginfo/meds/a601240-es.html

Sociedad Americana de Farmacéuticos Institucionales SA. (15 de Noviembre de 2016). MedlinePlus. Recuperado el 09 de Mayo de 2020, de https:// medlineplus.gov/spanish/druginfo/meds/a602015es.html

\section{CITAR ESTE ARTICULO:}

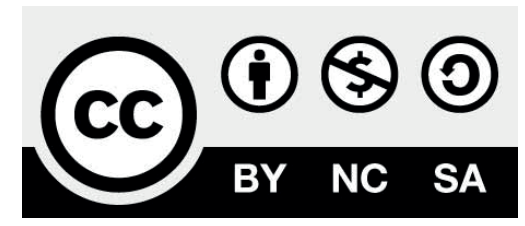

RECONOCIMIENTO-NOCOMERCIAL-COMPARTIRIGUAL CC BY-NC-SA

ESTA LICENCIA PERMITE A OTROS ENTREMEZCLAR, AJUSTAR Y CONSTRUIR A PARTIR DE SU OBRA CON FINES NO COMERCIALES, SIEMPRE Y CUANDO LE RECONOZCAN LA AUTORÍA Y SUS NUEVAS CREACIONES ESTÉN BAJO UNA LICENCIA CON LOS MISMOS TÉRMINOS.

Vargas Párraga, G., Chafla Pinduisaca, F., López Laaz, S., \& Correa Quinto, W. (2020). Recomendaciones de manejo farmacológico en COVID-19. REClMUNDO, 4(2), 31-39. doi:10.26820/recimundo/4.(2).mayo.2020.31-39 